\title{
Do Interventions with Diet or Dietary Supplements Reduce the Disease Activity Score in Rheumatoid Arthritis? A Systematic Review of Randomized Controlled Trials
}

\author{
Josefine Nelson ${ }^{1}$, Helen Sjöblom ${ }^{2}$, Inger Gjertsson ${ }^{3}$, Stine M. Ulven ${ }^{4}$, Helen M. Lindqvist ${ }^{5}$ (D) \\ and Linnea Bärebring ${ }^{5, *}$ \\ 1 The Department of Biosciences and Nutrition, Stockholm University, 17177 Stockholm, Sweden; \\ fysioterapi@perita.se \\ 2 Biomedical Library, Gothenburg University Library, University of Gothenburg, 40530 Gothenburg, Sweden; \\ helen.sjoblom@ub.gu.se \\ 3 Department of Rheumatology and Inflammation Research, Institute of Medicine, Sahlgrenska Academy, \\ University of Gothenburg, 40530 Gothenburg, Sweden; inger.gjertsson@rheuma.gu.se \\ 4 Department of Nutrition, Institute of Basic Medical Sciences, University of Oslo, Blindern, \\ 0317 Oslo, Norway; smulven@medisin.uio.no \\ 5 Department of Internal Medicine and Clinical Nutrition, University of Gothenburg, \\ 40530 Gothenburg, Sweden; helen.lindqvist@gu.se \\ * Correspondence: linnea.barebring@gu.se
}

Received: 29 June 2020; Accepted: 25 September 2020; Published: 29 September 2020

\begin{abstract}
The aim was to compile the evidence from Randomized Controlled Trials (RCTs) of diet or dietary supplements used to reduce disease activity in adults with Rheumatoid Arthritis (RA). Searches were performed in the databases PubMed, Scopus and Cochrane. Only RCT studies of diets, foods or dietary supplements, looking at effects on the Disease Activity Score in 28 joints (DAS28) among adults with RA, published in peer-reviewed journals, were included. A total of 27 articles were included - three of whole diets (Mediterranean diet, raw food and anti-inflammatory diet), five of food items, five of n-3 fatty acids, five of single micronutrient supplements, four of single antioxidant supplements and five of pre-, pro- or synbiotics. Studies that showed moderate strength evidence for positive effects on disease activity in RA included interventions with a Mediterranean diet, spices (ginger powder, cinnamon powder, saffron), antioxidants (quercetin and ubiquinone), and probiotics containing Lactobacillus Casei. Other diets or supplements had either no effects or low to very low strength of evidence. In conclusion, RCT studies on diet or dietary supplements are limited in patients with RA, but based on the results in this review there is evidence that some interventions might have positive effects on DAS28.
\end{abstract}

Keywords: Rheumatoid Arthritis; DAS28; diet

\section{Introduction}

Rheumatoid arthritis (RA) is a chronic autoimmune disease characterized by systemic inflammation and joint destruction. RA affects $0.5-1 \%$ of the western population and many patients have an active disease, despite pharmacological treatment [1]. Persistent disease activity causes the destruction of bone and cartilage, and negatively impacts quality of life due to pain, fatigue, depression and decreased function [2]. Many patients with RA report that their dietary intake affects their disease symptoms [3], and request dietary advice to complement their existing treatment [4]. However, there is a lack of evidence for dietary effects on disease activity in RA, and consequently no such dietary guidelines exist. 
Several nutrients and dietary components interact with the immune system and could affect disease activity in RA. These include omega-3 fatty acids, antioxidants [5], vitamin D [6], vitamin B6 [7], zinc and selenium [8]. In addition, pre-, pro- and synbiotics could have positive effects on RA $[9,10]$. The Mediterranean diet or Healthy Nordic Diet combine intakes of dietary fiber, antioxidants and omega-3 fatty acids through a high intake of fruit, berries, vegetable, whole grains, legumes, vegetable oil and fish [11,12], and could therefore be beneficial in RA. Other diets that could be beneficial are diets that are high in prebiotics in the form of dietary fiber, such as raw food [13] or vegan diets [14].

Despite indications that diet and dietary components could have positive effects on RA, few studies have summarized the results from randomized controlled trials (RCTs) in a systematic manner. A Cochrane review from 2009 [15] compiled the evidence for dietary interventions in RA, but excluded studies on dietary supplements. In addition, new studies have been published in the last decade, warranting an updated survey of the field. There is currently no fully encompassing, contemporary review that includes both diet and dietary supplements and their effect on disease activity in RA. The aim of this systematic review is therefore to compile and rate the evidence from RCTs of diet or dietary supplements used for reducing disease activity, measured by the commonly used composite instrument Disease Activity Score in 28 joints (DAS28) in adults with RA.

\section{Materials and Methods}

This systematic review was conducted and reported according to the Preferred Reporting Items for Systematic Review and Meta-Analyses Protocols (PRISMA) format [16] (Figure 1). The study protocol was registered in the International Prospective Register of Systematic Reviews (PROSPERO, no CRD42019141014). A Population, Intervention, Comparison and Outcomes (PICO) statement was used to define the research question "Can intervention with diet, food or dietary supplements reduce disease activity in RA?" (Table S1).

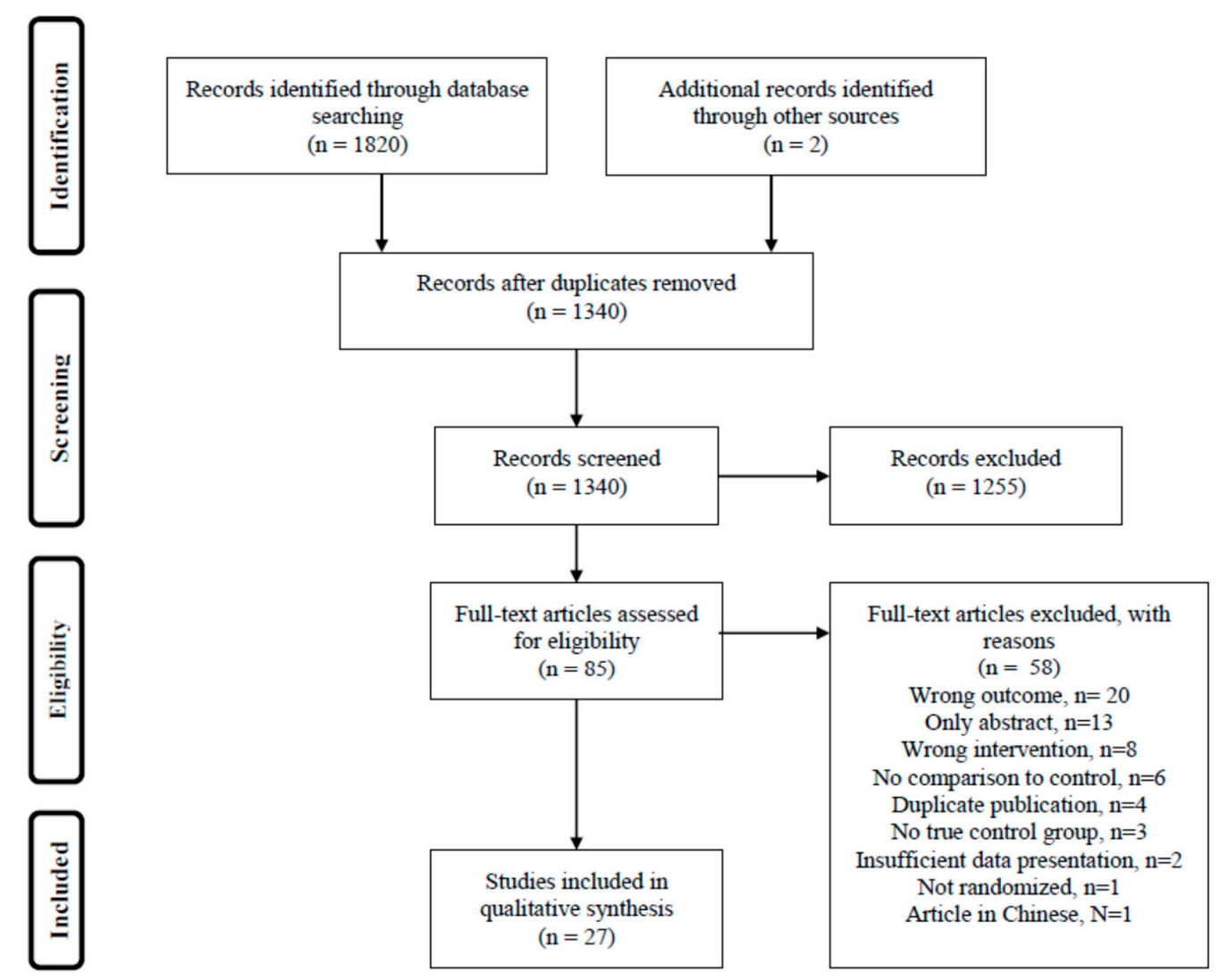

Figure 1. Flow chart of the article selection process according to the Preferred Reporting Items for Systematic Reviews and Meta-analyses (PRISMA). 
The inclusion criteria for this systematic review were articles that studied the following:

- $\quad$ Adults ( $\geq 18$ years) with RA;

- Randomized controlled trials - either parallel or crossover designs;

- Both or either diet or dietary supplements, including whole diet, specific foods, spices, herbs, nutrients, dietary antioxidants and pre-, pro- or synbiotics;

- Disease activity measured by DAS28 or DAS (see below) as an outcome of the intervention.

There were no limitations on study durations, dropout rates, participant gender or nutritional status. Dietary supplements were defined as formulations containing micronutrients, amino acids or fatty acids.

The exclusion criteria for this systematic review were as follows:

- Animal models;

- Observational studies;

- Non-randomized trials;

- Trials that lack a control group or regimen, including those comparing high dose to low dose;

- Results that were not peer reviewed, such as conference abstracts or publications in journals with no peer review process;

- Studies on natural remedies of a traditional medicinal type or herbal remedies, or food extracts;

- Studies that include other measures of disease activity than DAS/DAS28;

- Studies of other types of rheumatic diseases, including juvenile idiopathic arthritis.

For the clinical assessment of disease activity in RA, the most commonly used instrument is DAS28. DAS28 is a composite score that includes the status of 28 joints (tenderness and swelling), inflammation markers' erythrocyte sedimentation rate (ESR) or C-reactive protein (CRP) in combination with self-perceived health using a visual analogue scale (VAS). A DAS28 score $<2.6$ indicates remission, 2.6-3.2 low disease activity, 3.2-5.1 intermediate disease activity and $>5.1$ high disease activity [17].

\section{Data Collection and Processing}

The detailed search strategy is presented in Table S2. Searches (24 June 2019 and 21 March 2020) in the databases PubMed, Scopus and Cochrane library were performed by a trained librarian from the Biomedical Library at the University of Gothenburg, and delivered to the assessors in Rayyan. Two blinded assessors screened the abstracts, and articles that seemed to meet the criteria for inclusion were read in full length. Data were extracted using a pre-specified protocol. If data were presented from both intention to treat (ITT) and per-protocol analyses, both results were extracted but ITT was considered the primary analysis. If data on both DAS28-ESR and DAS28-CRP were presented, both results were extracted but DAS28-ESR was considered the main outcome variable.

The included articles were assessed in terms of quality using the Agency for Healthcare Research and Quality assessment tool for clinical trials according to the checklist by the Nordic Nutrition Recommendations 5 working group [18]. The individual studies were graded as A (considered high-quality with low risk of bias and likely valid results), B (moderate quality, with some risk of bias) or C (low-quality with significant risk of bias that may invalidate the results). After quality review, the studies were grouped by intervention type and a summary of evidence was given to each group, using GRADE for RCT studies [19]. According to GRADE, the evidence was graded as very low $(+)$, low $(++)$, moderate $(+++)$ or high $(++++)$. Each intervention type was assessed for problems with bias, consistency in results between trials, relevance, precision and risk of publication bias. No meta-analysis was attempted as the study designs and results were expected to be too heterogeneous. 


\section{Results}

The searches resulted in a total of 1340 individual articles. Two additional articles were identified from references used by other articles. After screening the abstracts, 85 articles were read in full length, and 27 met the inclusion criteria and were included. The remaining 58 studies were excluded based on wrong outcome, abstract only, insufficient data presentation, no comparison to control group, dual publishing, wrong intervention, no true control group, not randomized or language other than English or Scandinavian (Figure 1). Summaries of the 27 included studies are shown in Tables 1-3. Quality of evidence is summarized in Table 4.

\subsection{Studies on Whole Diets, Foods or Meals}

Three RCT studies investigated the effects of whole diets (Mediterranean diet, uncooked vegan diet, and Anti-inflammatory Mediterranean style diet) on DAS28; two parallel trials [20,21] and one crossover trial [22]. All studies were performed in the Nordic region (Sweden and Finland), but were performed and written by different researchers. Intervention diets were provided, in part or in full, to the participants in all three studies, but only the study by Vadell et al. provided the same amount of foods to the control group. The remaining two studies provided either no food [21] or food in the initial phase [20] to the control group. In two of the studies, the participants in the control group were instructed to follow their habitual diet, while in the study by Vadell et al., the participants received a control diet that was based on the national average intake. The number of participants randomized in the studies varied from 43 to 56. Both genders were included but women were in the majority. At study baseline, disease activity was intermediate for all three studies. Sköldstam et al. found a significant improvement in DAS28 (effects on tender joints and CRP) when comparing the Mediterranean diet intervention to control diet (habitual diet) after 12 weeks. Vadell et al. found tendencies towards positive effects after the 10 week Anti-inflammatory Mediterranean style diet intervention, but these were not significant in adjusted comparisons to the control diet [22]. Nenonen et al. found no effect on DAS28 from the 12 week raw food vegan diet intervention [21]. The quality of the studies was high for the study by Vadell et al., intermediate for the study by Sköldstam et al., and low for the study by Nenonen et al. In sum, the quality of evidence that a Mediterranean style diet reduced DAS28 in RA was considered moderate. The quality of evidence for effects of vegan, raw food diet was very low and too limited to draw any conclusions.

Two RCT studies investigated the effects of food items on disease activity-one parallel trial studying the intake of cranberry juice [23] and one crossover trial studying the intake of blue mussels [24]. The studies included 39 and 41 participants, all female. Lindqvist et al. compared the effect of one daily ready-to-eat meal ( 5 days/week) for 11 weeks, supplemented with either $75 \mathrm{~g}$ blue mussels or chicken/red meat [24]. Thimoteo et al. compared a usual diet supplemented with $500 \mathrm{~mL} / \mathrm{day}$ low calorie cranberry juice to a usual diet, for 3 months [23]. The baseline disease activity was intermediate in both studies. Thimoteo et al. found no significant effect of DAS28. However, Lindqvist et al. found a decrease in DAS28 in ITT analysis (effects on general health), but not in per protocol analysis. The quality of both studies was intermediate. The quality of evidence that a daily consumption of blue mussels reduces DAS28 in RA was considered low. Cranberry juice does not seem to reduce DAS28 in RA (moderate quality of evidence). 
Table 1. Randomized controlled studies on effects of diet, food and spices on Disease Activity Score in Rheumatoid Arthritis.

\begin{tabular}{|c|c|c|c|c|c|c|c|c|c|}
\hline $\begin{array}{l}\text { Author, Year, Country } \\
\text { [Ref] }\end{array}$ & Participants & Population & $\begin{array}{c}\text { Study } \\
\text { Duration }\end{array}$ & Intervention & Control & $\begin{array}{l}\text { DAS28 Main } \\
\text { Results }\end{array}$ & $\begin{array}{l}\text { Adjusted } \\
\text { Analysis }\end{array}$ & $\begin{array}{c}\text { Study } \\
\text { Quality }\end{array}$ & Comments \\
\hline $\begin{array}{l}\text { Sköldstam } 2012 \\
\text { Sweden } \\
\text { [20] } \\
\text { RCT UB }\end{array}$ & $\begin{array}{l}\text { N randomized: } 56 \\
\text { N control: } 27 \\
\text { N intervention: } 29 \\
\text { Total drop out: } 9 \%\end{array}$ & $\begin{array}{c}80 \% \text { women } \\
49 \% \text { MTX use } \\
\text { Disease activity: } \\
\text { Intermediate }\end{array}$ & 12 weeks & $\begin{array}{c}\text { Mediterranean diet } \\
\text { Foods provided: Some meals in } \\
\text { the initial } 3 \text { weeks. Margarine, } \\
\text { olive oil, canola oil, frozen } \\
\text { vegetables, tea. }\end{array}$ & $\begin{array}{c}\text { Usual diet } \\
\text { Foods provided: Some } \\
\text { meals in the initial } \\
3 \text { weeks }\end{array}$ & $\begin{array}{l}\text { DAS28 ESR } \\
\text { I: } 4.4 \rightarrow 3.9 \\
\text { C: } 4.3 \rightarrow 4.3 \\
P=0.047\end{array}$ & Unadjusted & B & $\begin{array}{l}\text { No relevant power calculation } \\
\text { Weight loss in intervention group } \\
\text { Study violations not excluded }\end{array}$ \\
\hline $\begin{array}{l}\text { Nenonen 1998Finland } \\
\text { [21] RCT UB }\end{array}$ & $\begin{array}{l}\text { N randomized: } 43 \\
\text { N control: } 21 \mathrm{~N} \\
\text { intervention: } 22 \\
\text { Total drop out: } 5 \%\end{array}$ & $\begin{array}{l}86 \% \text { women } \\
35 \% \text { MTX use } \\
\text { Disease activity: } \\
\text { Intermediate }\end{array}$ & 3 months & $\begin{array}{l}\text { Uncooked vegan diet } \\
\text { Foods provided: All foods }\end{array}$ & $\begin{array}{l}\text { Usual omnivore diet } \\
\text { Foods provided: None }\end{array}$ & $\begin{array}{c}\text { DAS28: } \\
\text { I: } 3.26 \rightarrow 3.01 \\
\text { C: } 3.44 \rightarrow 3.46 \\
\quad P=0.7\end{array}$ & $\begin{array}{l}\text { Adjusted for } \\
\text { weight change }\end{array}$ & c & $\begin{array}{l}\text { No relevant power calculation } \\
\text { Weight loss in intervention group } \\
\text { Calculation of DAS28 unclear }\end{array}$ \\
\hline $\begin{array}{c}\text { Vadell 2019Sweden } \\
{[22]} \\
\text { RCOT SB (assessors) }\end{array}$ & $\begin{array}{l}\text { N randomized: } 50 \\
\text { N control: } \\
\text { N intervention: } \\
\text { Total drop out: } 12 \%\end{array}$ & $\begin{array}{l}77 \% \text { women } \\
66 \% \text { MTX use } \\
\text { Disease activity: } \\
\text { Intermediate }\end{array}$ & 10 weeks & $\begin{array}{l}\text { Anti-inflammatory, } \\
\text { Mediterranean style diet, rich in } \\
\text { fruit, berries, vegetable, nuts, } \\
\text { whole grain, low fat dairy, } \\
\text { fish and vegetable oil } \\
\text { Foods provided: Breakfast, main } \\
\text { meal, one snack per day for } \\
5 \mathrm{~d} / \mathrm{w}\end{array}$ & $\begin{array}{l}\text { Average Swedish diet, } \\
\text { high in red meat, } \\
\text { refined grains, butter, } \\
\text { quark, protein bars } \\
\text { Foods provided: } \\
\text { Breakfast, main meal, } \\
\text { one snack per day for } \\
5 \mathrm{~d} / \mathrm{w}\end{array}$ & $\begin{array}{c}\text { DAS28 ESR: } \\
\text { I: } 3.39 \rightarrow 3.05 \\
\text { C: } 3.42 \rightarrow 3.27 \\
P=\text { NS }\end{array}$ & $\begin{array}{c}\text { Adjusted for } \\
\text { baseline, diet } \\
\text { sequence, } \\
\text { batch and } \\
\text { starting diet }\end{array}$ & A & $\begin{array}{l}\text { No drop out analysis but few } \\
\text { dropouts } \\
\text { Weight stable during both diets } \\
\text { Adjusted for baseline and carry } \\
\text { over effects }\end{array}$ \\
\hline $\begin{array}{c}\text { Thimoteo 2019Brazil } \\
\text { [23] } \\
\text { RCT UB }\end{array}$ & $\begin{array}{l}\text { N randomized: } 41 \mathrm{~N} \\
\text { intervention: } 23 \\
\quad \mathrm{~N} \text { control: } 18 \\
\text { Total drop out: } 7 \%\end{array}$ & $\begin{array}{l}100 \% \text { women } \\
63 \% \text { MTX use } \\
\text { Disease activity: } \\
\text { Intermediate }\end{array}$ & 3 months & $\begin{array}{l}\text { Usual diet plus } 500 \mathrm{~mL} / \mathrm{d} \text { of } \\
\text { reduced calorie cranberry juice }\end{array}$ & Usual diet & $\begin{array}{c}\text { DAS28 ESR: } \\
\text { I: } 3.48 \rightarrow 2.99 \\
\text { C: } 3.59 \rightarrow 3.52 \\
P=\text { NS }\end{array}$ & Unadjusted & B & $\begin{array}{l}\text { No drop out analysis but few } \\
\text { drop outs } \\
\text { Excluded those with poor } \\
\text { compliance } \\
\text { High drop out }\end{array}$ \\
\hline $\begin{array}{l}\text { Lindqvist 2018Sweden } \\
\text { [24] } \\
\text { RCOT SB (assessors) }\end{array}$ & $\begin{array}{l}\text { N randomized: } 39 \\
\text { N control: } 19 \\
\text { N intervention: } 20 \\
\text { Total drop out: } 41 \%\end{array}$ & $\begin{array}{l}100 \% \text { women } \\
60 \% \text { MTX use } \\
\text { Disease activity: } \\
\text { Intermediate }\end{array}$ & 11 weeks & $\begin{array}{l}5 \text { weekly meals with } 75 \mathrm{~g} \text { of blue } \\
\text { mussels in addition to } \\
\text { normal diet } \\
\text { Foods provided: all } 5 \text { meals/w }\end{array}$ & $\begin{array}{c}5 \text { weekly meals with } \\
\text { meat/chicken in } \\
\text { addition to normal diet } \\
\text { Foods provided: all } 5 \\
\text { meals/w }\end{array}$ & $\begin{array}{c}\text { DAS28 (ITT) } \\
\text { I: } 3.96 \rightarrow 3.40 \\
\text { C: } 3.95 \rightarrow 3.88 \\
\quad P=0.023\end{array}$ & Unadjusted & B & $\begin{array}{c}\text { Did not consider carry over } \\
\text { effects in analysis } \\
\text { NS effects on DAS28 ESR in PP } \\
\text { analysis } \\
\text { DAS28 CRP also lower after } \\
\text { intervention diet }\end{array}$ \\
\hline $\begin{array}{l}\text { Aryaeian } 2019 \\
\text { Iran } \\
{[25]} \\
\text { RCT DB }\end{array}$ & $\begin{array}{l}\text { N randomized: } 70 \mathrm{~N} \\
\text { intervention: } 35 \\
\mathrm{~N} \text { control: } 35 \\
\text { Total drop out: } 10 \%\end{array}$ & $\begin{array}{l}\text { 89\% women } \\
\text { 97\% MTX use } \\
\text { Disease activity: } \\
\text { Intermediate }\end{array}$ & 12 weeks & $\begin{array}{l}1500 \text { mg ginger powder in } 2 \\
\text { capsules daily }\end{array}$ & $\begin{array}{l}\text { Similar capsules with } \\
\text { wheat flour }\end{array}$ & $\begin{array}{l}\text { DAS28 ESR: } \\
\text { I: } 4.73 \rightarrow 3.44 \\
\text { C: } 4.51 \rightarrow 4.30 \\
P=0.003\end{array}$ & Unadjusted & B & $\begin{array}{l}\text { No relevant power calculation } \\
\text { No drop out analysis but few } \\
\text { drop outs }\end{array}$ \\
\hline $\begin{array}{l}\text { Shishehbor } 2018 \\
\text { Iran } \\
{[26]} \\
\text { RCT DB }\end{array}$ & $\begin{array}{l}\text { N randomized: } 40 \\
\text { N intervention: } 20 \\
\text { N control: } 20 \\
\text { Total drop out: } 10 \%\end{array}$ & $\begin{array}{c}\text { 100\% women } \\
78 \% \text { MTX use } \\
\text { Disease activity: } \\
\text { High }\end{array}$ & 8 weeks & $\begin{array}{l}2000 \mathrm{mg} \text { cinnamon powder in } 4 \\
\text { capsules daily }\end{array}$ & $\begin{array}{l}\text { Similar capsules with } \\
\text { starch }\end{array}$ & $\begin{array}{l}\text { DAS28: } \\
\text { I: } 6.04 \rightarrow 3.92 \\
\text { C: } 5.35 \rightarrow 5.64 \\
P<0.001\end{array}$ & $\begin{array}{l}\text { Adjusted for } \\
\text { baseline and } \\
\text { menopausal } \\
\text { status }\end{array}$ & A & Almost worse by control \\
\hline $\begin{array}{l}\text { Hamidi } \\
2020 \\
\text { Iran } \\
{[27]} \\
\text { RCT DB }\end{array}$ & $\begin{array}{l}\text { N randomized: } 66 \\
\text { N intervention: } 33 \\
\text { N control: } 33 \\
\text { Total drop out: } 8 \%\end{array}$ & $\begin{array}{l}100 \% \text { women } \\
\% \text { MTX use not } \\
\text { specified } \\
\text { Disease activity: } \\
\text { Intermediate }\end{array}$ & 3 months & $100 \mathrm{mg} / \mathrm{d}$ saffron in 1 tablet & $\begin{array}{l}\text { Placebo (100 mg } \\
\text { hydroxyl-propylmethyl } \\
\text { cellulose in } 1 \text { tablet) }\end{array}$ & $\begin{array}{c}\text { DAS28 CRP: } \\
\text { I: } 5.09 \rightarrow 4.33 \\
\text { C: } 4.92 \rightarrow 5.19 \\
P<0.001\end{array}$ & Unadjusted & B & $\begin{array}{c}\text { Almost worse by control } \\
\text { No drop out analysis but few } \\
\text { drop outs } \\
\text { Excluded uncompliant } \\
\text { participants, though few } \\
\text { Did not adjust for baseline, } \\
\text { despite slight imbalance }\end{array}$ \\
\hline
\end{tabular}

RCT; randomized controlled trial, RCOT; randomized crossover trial, UB; unblinded, SB; single blind, DB; double blind, N; number, MTX; metrotrexate, ITT; Intention to treat. 
Table 2. Randomized controlled studies of effects of single nutrients or antioxidants on Disease Activity Score in Rheumatoid Arthritis.

\begin{tabular}{|c|c|c|c|c|c|c|c|c|c|}
\hline $\begin{array}{l}\text { Author, Year, Country } \\
\text { (Ref) }\end{array}$ & Participants & Population & $\begin{array}{c}\text { Study } \\
\text { Duration }\end{array}$ & Intervention & Control & $\begin{array}{l}\text { DAS28 Main } \\
\text { Results }\end{array}$ & $\begin{array}{l}\text { Adjusted } \\
\text { Analysis }\end{array}$ & $\begin{array}{l}\text { Study } \\
\text { Quality }\end{array}$ & Comments \\
\hline $\begin{array}{c}\text { Galarraga 2008UK } \\
\text { (Scotland) } \\
{[28]} \\
\text { RCT DB }\end{array}$ & $\begin{array}{l}\text { N randomized: } 97 \mathrm{~N} \\
\text { intervention: } 49 \\
\text { N control: } 48 \\
\text { Total drop out: } 40 \%\end{array}$ & $\begin{array}{l}71 \% \text { women } \\
\text { 77\% DMARD use } \\
\text { Disease activity: } \\
\text { Intermediate }\end{array}$ & 12 weeks & 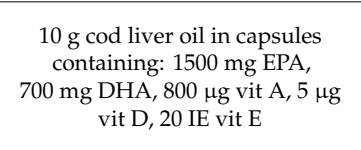 & $\begin{array}{l}\text { Placebo (air filled } \\
\text { capsules) }\end{array}$ & $\begin{array}{l}\text { DAS28 CRP: } \\
\text { I: } 4.5 \rightarrow 4.3 \\
\text { C: } 4.5 \rightarrow 4.3 \\
P=0.976\end{array}$ & Unadjusted & B & $\begin{array}{l}\text { Study duration was } 9 \text { mo but } \\
\text { after } 12 \mathrm{w} \text { NSAID was reduced } \\
\text { Amount of n } 3 / \text { d unclear } \\
\text { No drop out analysis } \\
\text { High drop out }\end{array}$ \\
\hline $\begin{array}{l}\text { Das Gupta } \\
\text { 2009 } \\
\text { Bangladesh } \\
\text { [29] RCT UB }\end{array}$ & $\begin{array}{l}\text { N randomized: } 100 \mathrm{~N} \\
\text { intervention: } 50 \\
\text { N control: } 50 \\
\text { Total drop out: } 19 \%\end{array}$ & $\begin{array}{l}\% \text { of women not } \\
\text { specified } \\
\% \text { MTX use not } \\
\text { specified } \\
\text { Disease activity: } \\
\text { High }\end{array}$ & 12 weeks & $\begin{array}{l}75 \mathrm{mg} / \mathrm{d} \text { indomethacin } \\
3 \mathrm{~g} / \mathrm{d} \mathrm{n} 3 \mathrm{FA} \text { in capsules }\end{array}$ & $75 \mathrm{mg} / \mathrm{d}$ indomethacin & $\begin{array}{l}\text { DAS28: } \\
\text { I: } 7.2 \rightarrow 4.2 \\
\text { C: } 7.3 \rightarrow 4.8 \\
\quad P<0.05\end{array}$ & Unadjusted & B & $\begin{array}{l}\text { No clear aim or hypothesis } \\
\text { No clear description of } \\
\text { participant characteristics } \\
\text { Calculation of DAS28 unclear }\end{array}$ \\
\hline $\begin{array}{l}\text { Dawczynski } \\
2018 \\
\text { Germany } \\
{[30]} \\
\text { RCOT DB }\end{array}$ & $\begin{array}{l}\mathrm{N} \text { randomized: } 38 \mathrm{~N} \\
\text { intervention: } 38 \\
\mathrm{~N} \text { control: } 38 \\
\text { Total drop out: } 34 \%\end{array}$ & $\begin{array}{l}84 \% \text { women } \\
\% \text { MTX use not } \\
\text { specified } \\
\text { Disease activity: } \\
\text { Intermediate }\end{array}$ & 10 weeks & $\begin{array}{l}8 \mathrm{~g} \text { micro algae, enriched in } 60 \mathrm{~g} \\
\text { sausage, } 8 \mathrm{~g} \text { tomato spread, } 30 \mathrm{~g} \\
\text { milk powder } \\
\text { Amount of n3/d: } 2.36 \mathrm{~g}\end{array}$ & $\begin{array}{c}8 \mathrm{~g} \text { sun flower oil, } \\
\text { enriched in } 60 \mathrm{~g} \\
\text { sausage, } 8 \mathrm{~g} \text { tomato } \\
\text { spread, } 30 \mathrm{~g} \text { milk } \\
\text { powder }\end{array}$ & $\begin{array}{c}\text { DAS28: } \\
\text { I: } 4.5 \rightarrow 3.88 \\
\text { C: } 3.99 \rightarrow 4.13 \\
P=0.085\end{array}$ & $\begin{array}{l}\text { Adjusted for } \\
\text { sequence and } \\
\text { baseline value }\end{array}$ & B & $\begin{array}{l}\text { Excluded those with poor } \\
\text { compliance } \\
\text { Calculation of DAS28 unclear }\end{array}$ \\
\hline $\begin{array}{l}\text { Dawczynski } \\
2009 \\
\text { Germany } \\
\text { [31] } \\
\text { RCOT UB }\end{array}$ & $\begin{array}{l}\mathrm{N} \text { randomized: } 45 \mathrm{~N} \\
\text { intervention: } 45 \\
\mathrm{~N} \text { control: } 45 \\
\text { Total drop out: } 13 \%\end{array}$ & $\begin{array}{l}96 \% \text { women } \\
\% \text { MTX use not } \\
\text { specified } \\
\text { Disease activity: } \\
\text { Intermediate }\end{array}$ & 12 weeks & $\begin{array}{l}40 \mathrm{~g} \text { fat in the form of } 200 \mathrm{~g} \\
\text { yoghurt, } 30 \mathrm{~g} \text { cheese and butter. } \\
\text { Milk fat was in part exchanged } \\
\text { with oils high in EPA, DHA and } \\
\text { alpha linoleic acid. } \\
\text { Amount of n } 3 / \mathrm{d}: 2.4 \mathrm{~g}\end{array}$ & $\begin{array}{l}\text { Commercial dairy } \\
\text { products with similar } \\
\text { fat content }\end{array}$ & $\begin{array}{c}\text { DAS28: } \\
\text { I: } 4.45 \rightarrow 4.32 \\
\text { C: } 4.18 \rightarrow 4.24 \\
P=\text { NS }\end{array}$ & $\begin{array}{l}\text { Adjusted for } \\
\text { sequence and } \\
\text { baseline value }\end{array}$ & B & $\begin{array}{c}\text { Excluded those with poor } \\
\text { compliance } \\
\text { Adjusted for potential carry over } \\
\text { effects in analysis } \\
\text { Calculation of DAS28 unclear } \\
\text { No relevant power calculation }\end{array}$ \\
\hline $\begin{array}{l}\text { Remans2004 } \\
\text { Netherlands } \\
\text { [32] RCT DB }\end{array}$ & $\begin{array}{l}\mathrm{N} \text { randomized: } 66 \mathrm{~N} \\
\text { intervention: } 33 \\
\mathrm{~N} \text { control: } 33 \\
\text { Total drop out: } 17 \%\end{array}$ & $\begin{array}{c}82 \% \text { women } \\
62 \% \text { MTX use } \\
\text { Disease activity: } \\
\text { High }\end{array}$ & 4 months & $\begin{array}{l}\text { Liquid nutritional supplement } \\
\text { containing PUFA (1400 mg EPA } \\
211 \mathrm{mg} \text { DHA, } 40 \mathrm{mg} \text { DPA, } 16 \mathrm{mg} \\
\text { ALA) and micronutrients }\end{array}$ & $\begin{array}{l}\text { Placebo drink with the } \\
\text { same taste, odor and } \\
\text { color but with artificial } \\
\text { sweetener }\end{array}$ & $\begin{array}{c}\text { DAS28: } \\
\text { I: } 5.36 \rightarrow 5.58 \\
\text { C: } 5.14 \rightarrow 5.35 \\
\quad P=\text { NS }\end{array}$ & Unadjusted & B & $\begin{array}{l}\text { Slight gain in body weight in } \\
\text { intervention group } \\
\text { Calculation of DAS28 unclear } \\
\text { No drop out analysis }\end{array}$ \\
\hline $\begin{array}{c}\text { Buondonno } 2017 \text { Italy } \\
\text { [33] } \\
\text { RCT DB }\end{array}$ & $\begin{array}{l}\text { N randomized: } 39 \\
\text { N intervention: } 21 \\
\quad \text { control: } 18 \\
\text { Total drop out: } 8 \%\end{array}$ & $\begin{array}{c}\text { 100\% women } \\
\text { 100\% MTX use } \\
\text { Disease activity: } \\
\text { High }\end{array}$ & 3 months & $\begin{array}{l}300000 \mathrm{IU}(7500 \mu \mathrm{g}) \text { of vitamin } \\
\text { D3 administered once }\end{array}$ & Placebo & $\begin{array}{c}\text { DAS28 ESR: } \\
\text { I: } ? \rightarrow 5.6 \\
\text { C: } ? \rightarrow 5.8 \\
P=\mathrm{NS}\end{array}$ & Unadjusted & B & $\begin{array}{l}\text { Unclear DAS28 at baseline } \\
\text { No dropout analysis, but low } \\
\text { drop out }\end{array}$ \\
\hline $\begin{array}{c}\text { Hansen } 2014 \\
\text { USA } \\
\text { [34] } \\
\text { RCT DB }\end{array}$ & $\begin{array}{l}\text { N randomized: } 22 \\
\text { N intervention: } 11 \\
\text { N control: } 11 \\
\text { Total drop out: } 0 \%\end{array}$ & $\begin{array}{l}46 \% \text { women } \\
\% \text { MTX use not } \\
\text { specified } \\
\text { Disease activity: } \\
\text { Low }\end{array}$ & 12 months & $\begin{array}{c}\text { Month 1: 3*50 } 000 \mathrm{IU} \text { vitamin } \\
\text { D2/week } \\
\text { Month 2-11: 2*50 000 IU vitamin } \\
\text { D2/month } \\
1500 \mathrm{mg} \text { calcium daily }\end{array}$ & $\begin{array}{c}\text { Placebo } \\
1500 \text { mg calcium daily }\end{array}$ & $\begin{array}{c}\text { DAS28: } \\
\text { I: } 3.0 \rightarrow 3.03 \\
\text { C: } 2.54 \rightarrow 2.96 \\
P=\mathrm{NS}\end{array}$ & Unadjusted & C & $\begin{array}{l}\text { Sparse methods and results } \\
\text { No relevant power calculation } \\
\text { Calculation of DAS28 not } \\
\text { presented }\end{array}$ \\
\hline $\begin{array}{c}\text { Rastmanesh } 2008 \\
\text { Iran } \\
\text { [35] } \\
\text { RCT DB }\end{array}$ & $\begin{array}{l}\mathrm{N} \text { randomized: } 38 \mathrm{~N} \\
\text { intervention: } 18 \\
\mathrm{~N} \text { control: } 18 \\
\text { Total drop out: } 16 \%\end{array}$ & $\begin{array}{c}100 \% \text { women } \\
100 \% \text { DMARD } \\
\text { use } \\
\text { Disease activity: } \\
\text { High }\end{array}$ & 28 days & $\begin{array}{l}6000 \mathrm{mg} \text { of potassium in the form } \\
\text { of enriched white grape juice }\end{array}$ & Placebo grape juice & $\begin{array}{c}\text { DAS28: } \\
\text { I: } 5.86 \rightarrow-0.69 \\
\text { C: } 5.80 \rightarrow-0.1 \\
P<0.01\end{array}$ & $\begin{array}{l}\text { Adjusted for } \\
\text { baseline }\end{array}$ & B & $\begin{array}{l}\text { Hypokalemic participants } \\
\text { Short study duration } \\
\text { Calculation of DAS28 unclear } \\
\text { Compliance unclear } \\
\text { Conflict of interest statement } \\
\text { missing }\end{array}$ \\
\hline $\begin{array}{l}\text { Shishavan } 2015 \\
\text { Iran } \\
{[36]} \\
\text { RCT DB }\end{array}$ & $\begin{array}{l}\mathrm{N} \text { randomized: } 64 \mathrm{~N} \\
\text { intervention: } 32 \\
\mathrm{~N} \text { control: } 32 \\
\text { Total drop out: } 9 \%\end{array}$ & $\begin{array}{l}100 \% \text { women } \\
91 \% \text { MTX use } \\
\text { Disease activity: } \\
\text { Remission }\end{array}$ & 8 weeks & $\begin{array}{c}10 \mu \mathrm{\mu g} / \text { day of vitamin } \mathrm{K} 1 \text { as a } \\
\text { chewable tablet }\end{array}$ & Placebo & $\begin{array}{l}\text { DAS28 CRP: } \\
\text { I: } 1.74 \rightarrow 1.59 \\
\text { C: } 2.26 \rightarrow 1.85 \\
P=\text { NS }\end{array}$ & $\begin{array}{l}\text { Adjusted } \\
\text { (baseline, } \\
\text { duration, folic } \\
\text { acid intake, } \\
\text { energy intake } \\
\text { and weight) }\end{array}$ & A & $\begin{array}{l}\text { No drop out analysis, but few } \\
\text { drop outs } \\
\text { Participants in remission at } \\
\text { baseline }\end{array}$ \\
\hline
\end{tabular}


Table 2. Cont.

\begin{tabular}{|c|c|c|c|c|c|c|c|c|c|}
\hline $\begin{array}{l}\text { Author, Year, Country } \\
\text { (Ref) }\end{array}$ & Participants & Population & $\begin{array}{l}\text { Study } \\
\text { Duration }\end{array}$ & Intervention & Control & $\begin{array}{l}\text { DAS28 Main } \\
\text { Results }\end{array}$ & $\begin{array}{l}\text { Adjusted } \\
\text { Analysis }\end{array}$ & $\begin{array}{l}\text { Study } \\
\text { Quality }\end{array}$ & Comments \\
\hline $\begin{array}{c}\text { Van Ede } 2001 \\
\text { Netherlands } \\
\text { [37] } \\
\text { RCT DB }\end{array}$ & $\begin{array}{c}\text { N randomized: } 434 \mathrm{~N} \\
\text { intervention 1: } 143 \\
\text { N intervention 2: } 147 \\
\text { N control: } 144 \\
\text { Total drop out unclear }\end{array}$ & $\begin{array}{l}71 \% \text { women } \\
\text { 100\% MTX use } \\
\text { Disease activity: } \\
\text { Intermediate }\end{array}$ & 12 months & $\begin{array}{l}\text { Intervention } 1: 1 \mathrm{mg} / \text { day of folic } \\
\text { acid (oral, intake in morning) } \\
\text { Intervention 2: } 2.5 \mathrm{mg} / \mathrm{week} \text { of } \\
\text { folinic acid (oral, within } 24 \mathrm{~h} \text { of } \\
\text { MTX intake) }\end{array}$ & Placebo & $\begin{aligned} \text { DAS28 ESR: } \\
\text { I 1: } 4.8 \rightarrow-1.5 \\
\text { I } 2: 4.6 \rightarrow-1.4 \\
\text { C: } 4.7 \rightarrow-1.5 \\
\text { P1 }=\text { NS } \\
\text { P2 }=\text { NS }\end{aligned}$ & Unadjusted? & B & $\begin{array}{l}\text { Drop out unclear } \\
\text { Unclear if analysis is adjusted }\end{array}$ \\
\hline $\begin{array}{l}\text { Gargari } \\
2015 \\
\text { Iran } \\
{[38]} \\
\text { RCT DB }\end{array}$ & $\begin{array}{l}\mathrm{N} \text { randomized: } 70 \mathrm{~N} \\
\text { intervention: } 35 \\
\mathrm{~N} \text { control: } 35 \\
\text { Total drop out: } 7 \%\end{array}$ & $\begin{array}{l}100 \% \text { women } \\
88 \% \text { MTX use } \\
\text { Disease activity: } \\
\text { Remission }\end{array}$ & 8 weeks & $\begin{array}{l}\text { Two capsules of } 1200 \mathrm{mg} \text { alpha } \\
\text { lipoic acid }\end{array}$ & $\begin{array}{l}\text { Placebo (1200 mg } \\
\text { maltodextrin) }\end{array}$ & $\begin{array}{c}\text { DAS28: } \\
\text { I: } 2.11 \rightarrow 1.86 \\
\text { C: } 2.14 \rightarrow 1.98 \\
P=0.442\end{array}$ & $\begin{array}{l}\text { Adjusted for } \\
\text { baseline value }\end{array}$ & A & $\begin{array}{c}\text { Excluded those with poor } \\
\text { compliance, but few } \\
\text { No dropout analysis, but few } \\
\text { dropouts } \\
\text { Participants in remission at } \\
\text { baseline } \\
\text { Calculation of DAS28 unclear }\end{array}$ \\
\hline $\begin{array}{l}\text { Javadi } 2016 \\
\text { Iran } \\
\text { [39] RCT DB }\end{array}$ & $\begin{array}{l}\mathrm{N} \text { randomized: } 50 \mathrm{~N} \\
\text { intervention: } 25 \\
\mathrm{~N} \text { control: } 25 \\
\text { Total drop out: } 20 \%\end{array}$ & $\begin{array}{c}\text { 100\% women } \\
\text { 92\% MTX use } \\
\text { Disease activity: } \\
\text { Low-intermediate }\end{array}$ & 8 weeks & $\begin{array}{l}\text { One capsule containing } 500 \mathrm{mg} \\
\text { quercetin }\end{array}$ & Placebo (lactose) & $\begin{array}{c}\text { DAS28 ESR: } \\
\text { I: } 3.22 \rightarrow 2.65 \\
\text { C: } 3.13 \rightarrow 3.11 \\
P=0.04\end{array}$ & $\begin{array}{c}\text { Adjusted for } \\
\text { baseline value }\end{array}$ & B & $\begin{array}{c}\text { Conflict of interest statement } \\
\text { missing } \\
\text { No drop out analysis } \\
\text { Excluded those with poor } \\
\text { compliance }\end{array}$ \\
\hline $\begin{array}{l}\text { Nachvak } 2019 \\
\text { Iran } \\
{[40]} \\
\text { RCT DB }\end{array}$ & $\begin{array}{l}\mathrm{N} \text { randomized: } 54 \mathrm{~N} \\
\text { intervention: } 27 \\
\mathrm{~N} \text { control: } 27 \\
\text { Total drop out: } 17 \%\end{array}$ & $\begin{array}{l}\text { 89\% women } \\
\% \text { MTX use not } \\
\text { specified } \\
\text { Disease activity: } \\
\text { Intermediate }\end{array}$ & 2 months & $100 \mathrm{mg} / \mathrm{day}$ CoQ10 capsules & Placebo & $\begin{array}{l}\text { DAS28 ESR: } \\
\text { I: } 5.01 \rightarrow 2.34 \\
\text { C: } 4.88 \rightarrow 4.04 \\
P<0.001\end{array}$ & $\begin{array}{c}\text { Adjusted for } \\
\text { baseline, age, } \\
\text { sex, disease } \\
\text { duration, } \\
\text { medications, } \\
\text { and total } \\
\text { energy intake }\end{array}$ & B & $\begin{array}{l}\text { Compliance unclear } \\
\text { No drop out analysis } \\
\text { Placebo unclear }\end{array}$ \\
\hline $\begin{array}{l}\text { Khojah } 2018 \\
\text { Egypt } \\
\text { [41] } \\
\text { RCT UB }\end{array}$ & $\begin{array}{l}\text { N randomized: } 100 \mathrm{~N} \\
\text { intervention: } 50 \\
\quad \text { control: } 50 \\
\text { Total drop out: } 0 \%\end{array}$ & $\begin{array}{l}68 \% \text { women } \\
\% \text { MTX use not } \\
\text { specified } \\
\text { Disease activity: } \\
\text { Intermediate }\end{array}$ & 3 months & $\begin{array}{l}\text { One capsule containing } 1 \mathrm{~g} \\
\text { resveratrol daily }\end{array}$ & Regular treatment & $\begin{array}{c}\text { DAS28 ESR: } \\
\text { I: } 4.62 \rightarrow 3.12 \\
\text { C: } 4.91 \rightarrow 4.78 \\
P<0.001\end{array}$ & Unadjusted & B & $\begin{array}{l}\text { No placebo } \\
\text { No relevant power analysis } \\
\text { Compliance unclear }\end{array}$ \\
\hline
\end{tabular}

RCT; randomized controlled trial, RCOT; randomized crossover trial, UB; unblinded, SB; single blind, DB; double blind, N; number, MTX; metrotrexate, ITT; Intention to treat. 
Table 3. Randomized controlled studies of effects of pre-, pro- or synbiotics on Disease Activity Score in Rheumatoid Arthritis

\begin{tabular}{|c|c|c|c|c|c|c|c|c|c|}
\hline $\begin{array}{l}\text { Author, Year, Country } \\
\text { [Ref] }\end{array}$ & Participants & Population & $\begin{array}{c}\text { Study } \\
\text { Duration }\end{array}$ & Intervention & Control & $\begin{array}{l}\text { DAS28 Main } \\
\text { Results }\end{array}$ & $\begin{array}{l}\text { Adjusted } \\
\text { Analysis }\end{array}$ & $\begin{array}{c}\text { Study } \\
\text { Quality }\end{array}$ & Comments \\
\hline $\begin{array}{l}\text { Alavi } 2011 \\
\text { UK } \\
\text { [42] } \\
\text { RCT DB }\end{array}$ & $\begin{array}{l}\text { N randomized: } 78 \mathrm{~N} \\
\text { intervention: } 33 \\
\text { N control: } 36 \\
\text { Total drop out: } 22 \%\end{array}$ & $\begin{array}{l}81 \% \text { women } \\
\% \text { MTX use not } \\
\text { specified } \\
\text { Disease activity: } \\
\text { Intermediate }\end{array}$ & 6 months & $\begin{array}{l}\text { Dietary fiber supplement, } \\
\text { (Ambrotose complex: aloe vera } \\
\text { gel extract, arabinogalactan, gum } \\
\text { ghatti, gum tragacanth, } \\
\text { glucosamine) }-1,3 \mathrm{~g} / \text { day }\end{array}$ & Placebo (rice flour) & $\begin{array}{c}\text { DAS28: } \\
\text { I: } \sim 4.1 \rightarrow 4.0 \\
\text { C: } \sim 4.3 \rightarrow 3.8 \\
P=0.009\end{array}$ & Unadjusted & C & $\begin{array}{c}\text { No drop out analysis, but ITT } \\
\text { Baseline DAS28 and treatment } \\
\text { unavailable } \\
\text { Calculation of DAS28 unclear }\end{array}$ \\
\hline $\begin{array}{l}\text { Alipour } 2014 \\
\text { Iran } \\
\text { [43] } \\
\text { RCT DB }\end{array}$ & $\begin{array}{l}\text { N randomized: } 60 \mathrm{~N} \\
\text { intervention: } 30 \\
\text { N control: } 30 \\
\text { Total drop out: } 23 \%\end{array}$ & $\begin{array}{l}\text { 100\% women } \\
76 \% \text { MTX use } \\
\text { Disease activity: } \\
\text { Remisson }\end{array}$ & 8 weeks & $\begin{array}{l}\text { One daily capsule of L. casei } 01 \\
\text { min } 10^{8} \text { colony forming units } \\
\text { (plus maltrodextrin) }\end{array}$ & Placebo (maltrodextrin) & $\begin{array}{c}\text { DAS28 CRP } \\
\text { I: } 2.56 \rightarrow 2.07 \\
\text { C: } 2.31 \rightarrow 2.23 \\
P=0.039\end{array}$ & $\begin{array}{c}\text { Adjusted } \\
\text { (baseline, BMI } \\
\text { change, } \\
\text { anxiety and } \\
\text { menopausal } \\
\text { status) }\end{array}$ & B & $\begin{array}{l}\text { Excluded those with poor } \\
\text { compliance } \\
\text { No drop out analysis } \\
\text { Remission at baseline }\end{array}$ \\
\hline $\begin{array}{l}\text { Zamani } 2016 \\
\text { Iran } \\
{[44]} \\
\text { RCT DB }\end{array}$ & $\begin{array}{l}\mathrm{N} \text { randomized: } 60 \mathrm{~N} \\
\text { intervention: } 30 \\
\mathrm{~N} \text { control: } 30 \\
\text { Total drop out: } 0 \%\end{array}$ & $\begin{array}{l}\text { 85\% women } \\
\text { 97\% MTX use } \\
\text { Disease activity: } \\
\text { Intermediate }\end{array}$ & 8 weeks & $\begin{array}{c}\text { Probiotic capsules containing } \\
\text { L. acidophilus }\left(2 \times 10^{9} \mathrm{CFU}\right), \\
\text { L. casei }\left(2 \times 10^{9} \mathrm{CFU}\right), \text { B. bifidum } \\
\left.2 \times 10^{9} \mathrm{CFU}\right)\end{array}$ & Placebo (starch) & $\begin{array}{l}\text { DAS28: } \\
\text { I: } 4.0 \rightarrow 3.7 \\
\text { C: } 4.1 \rightarrow 4.0 \\
P=0.01\end{array}$ & $\begin{array}{l}\text { Adjusted for } \\
\text { baseline, age, } \\
\text { BMI }\end{array}$ & A & Calculation of DAS28 unclear \\
\hline $\begin{array}{c}\text { De Los Angeles Pineda } \\
2011 \\
\text { Canada } \\
{[45]} \\
\text { RCT DB }\end{array}$ & $\begin{array}{l}\text { N randomized: } 29 \mathrm{~N} \\
\text { intervention: } 15 \\
\mathrm{~N} \text { control: } 14 \\
\text { Total drop out: } 10 \%\end{array}$ & $\begin{array}{l}93 \% \text { women } \\
76 \% \text { MTX use } \\
\text { Disease activity: } \\
\text { Intermediate }\end{array}$ & 3 months & $\begin{array}{l}\text { Two daily capsules of } \\
\text { L. rhamnosus GR-1 and } \\
\text { L. reuteri RC-14 (each } 2 \text { billion } \\
\text { CFU), plus dextrose, potato } \\
\text { starch, microcrystalline cellulose } \\
\text { and magnesium stearate }\end{array}$ & $\begin{array}{l}\text { Placebo (dextrose, } \\
\text { potato starch, } \\
\text { microcrystalline } \\
\text { cellulose and } \\
\text { magnesium stearate) }\end{array}$ & $\begin{array}{c}\text { DAS } \\
\text { I: } 4.18 \rightarrow \\
\Delta-2.1 \\
\text { C: } 4.83 \rightarrow \\
\Delta-2.9 \\
P=0.77\end{array}$ & Unadjusted & B & $\begin{array}{l}\text { No power calculationNo drop } \\
\text { out analysis but few drop outs } \\
\text { Compliance unclear }\end{array}$ \\
\hline $\begin{array}{l}\text { Zamani } 2017 \\
\text { Iran } \\
{[46]} \\
\text { RCT DB }\end{array}$ & $\begin{array}{l}\text { N randomized: } 54 \mathrm{~N} \\
\text { intervention: } 27 \\
\mathrm{~N} \text { control: } 27 \\
\text { Total drop out: } 0 \%\end{array}$ & $\begin{array}{l}\text { 85\% women } \\
\text { 96\% MTX } \\
\text { Disease activity: } \\
\text { Intermediate }\end{array}$ & 8 weeks & $\begin{array}{l}\text { Synbiotic capsules containing } \\
\text { L. acidophilus, L. casei, B. } \\
\text { bifidum (each } 2 \times 10^{9} \text { CFU), } \\
\text { and } 800 \mathrm{mg} \text { inulin }\end{array}$ & Placebo (starch) & $\begin{array}{l}\text { DAS28: } \\
\text { I: } 4.2 \rightarrow 2.6 \\
\text { C: } 3.5 \rightarrow 3.2 \\
P<0.001\end{array}$ & $\begin{array}{l}\text { Adjusted for } \\
\text { baseline, BMI } \\
\text { and age }\end{array}$ & A & Calculation of DAS28 unclear \\
\hline
\end{tabular}

forming units, L; Lactobacillus, B; Bifidobacteria. 
Table 4. Summary of quality of evidence for effects of diet, food and dietary supplements on Disease Activity Score in patients with Rheumatoid Arthritis.

\begin{tabular}{|c|c|c|c|c|c|}
\hline Type of Intervention & Ref. & No Studies & Results for DAS28 & Quality of Evidence & Comments \\
\hline \multicolumn{6}{|l|}{ Whole Diet Interventions } \\
\hline 7Mediterranean style diet & {$[20,22]$} & 2 & $\begin{array}{l}\text { Inconsistent, but } \\
\text { suggestive }\end{array}$ & Moderate $(+++)$ & $\begin{array}{c}\text { Risk of bias: } 0 \\
\text { Consistency: }-1 \\
\text { Relevance: } 0 \\
\text { Precision: } 0 \\
\text { Publication } \\
\text { bias: } 0\end{array}$ \\
\hline Raw food diet & [21] & 1 & $\begin{array}{l}\text { Non-significant } \\
\text { compared to control }\end{array}$ & Very low (+) & $\begin{array}{c}\text { Risk of bias: }-2 \\
\text { Consistency: } 0 \\
\text { Relevance: }-1 \\
\text { Precision: } 0 \\
\text { Publication } \\
\text { bias: } 0\end{array}$ \\
\hline \multicolumn{6}{|l|}{ Single food items } \\
\hline Blue mussels & [24] & 1 & $\begin{array}{l}\text { Improvement } \\
\text { compared to control }\end{array}$ & Low $(++)$ & $\begin{array}{l}\text { Risk of bias: }-1 \\
\text { Consistency: } 0 \\
\text { Relevance: }-1 \\
\text { Precision: } 0 \\
\text { Publication } \\
\text { bias: } 0\end{array}$ \\
\hline Cranberry juice & [23] & 1 & $\begin{array}{l}\text { Non-significant } \\
\text { compared to control }\end{array}$ & Moderate $(+++)$ & $\begin{array}{c}\text { Risk of bias: }-1 \\
\text { Consistency: } 0 \\
\text { Relevance: } 0 \\
\text { Precision: } 0 \\
\text { Publication } \\
\text { bias: } 0\end{array}$ \\
\hline Ginger powder & [25] & 1 & $\begin{array}{l}\text { Improvement } \\
\text { compared to control }\end{array}$ & Moderate $(+++)$ & $\begin{array}{c}\text { Risk of bias: }-1 \\
\text { Consistency: } 0 \\
\text { Relevance: } 0 \\
\text { Precision: } 0 \\
\text { Publication } \\
\text { bias: } 0\end{array}$ \\
\hline Cinnamon powder & [26] & 1 & $\begin{array}{l}\text { Improvement } \\
\text { compared to control }\end{array}$ & Moderate $(+++)$ & $\begin{array}{c}\text { Risk of bias: } 0 \\
\text { Consistency: } 0 \\
\text { Relevance: } 0 \\
\text { Precision: }-1 \\
\text { Publication } \\
\text { bias: } 0\end{array}$ \\
\hline Saffron & [27] & 1 & $\begin{array}{l}\text { Improvement } \\
\text { compared to control }\end{array}$ & Moderate $(+++)$ & $\begin{array}{c}\text { Risk of bias: }-1 \\
\text { Consistency: } 0 \\
\text { Relevance: } 0 \\
\text { Precision: } 0 \\
\text { Publication } \\
\text { bias: } 0\end{array}$ \\
\hline \multicolumn{6}{|l|}{ Nutrients } \\
\hline $\mathrm{n}-3$ supplementation & [28-32] & 5 & $\begin{array}{l}\text { Inconsistent, most } \\
\text { studies show no effect }\end{array}$ & Low $(++)$ & $\begin{array}{l}\text { Risk of bias: }-1 \\
\text { Consistency: }-1 \\
\text { Relevance: } 0 \\
\text { Precision: } 0 \\
\text { Publication } \\
\text { bias: } 0\end{array}$ \\
\hline $\begin{array}{c}\text { Vitamin D } \\
\text { supplementation }\end{array}$ & {$[33,34]$} & 2 & $\begin{array}{l}\text { Consistently no } \\
\text { improvement } \\
\text { compared to control }\end{array}$ & Low $(++)$ & $\begin{array}{c}\text { Risk of bias: }-1 \\
\text { Consistency: } 0 \\
\text { Relevance: } 0 \\
\text { Precision: }-1 \\
\text { Publication } \\
\text { bias: } 0\end{array}$ \\
\hline
\end{tabular}


Table 4. Cont.

\begin{tabular}{|c|c|c|c|c|c|}
\hline Type of Intervention & Ref. & No Studies & Results for DAS28 & Quality of Evidence & Comments \\
\hline $\begin{array}{c}\text { Vitamin K } \\
\text { supplementation }\end{array}$ & [36] & 1 & $\begin{array}{l}\text { No improvement } \\
\text { compared to control }\end{array}$ & Low $(++)$ & $\begin{array}{l}\text { Risk of bias: } 0 \\
\text { Consistency: } 0 \\
\text { Relevance: }-1 \\
\text { Precision: }-1 \\
\text { Publication } \\
\text { bias: } 0\end{array}$ \\
\hline \multicolumn{6}{|l|}{ Single antioxidants } \\
\hline Alpha lipoic acid & {$[38]$} & 1 & $\begin{array}{l}\text { No improvement } \\
\text { compared to control }\end{array}$ & Moderate $(+++)$ & $\begin{array}{l}\text { Risk of bias: } 0 \\
\text { Consistency: } 0 \\
\text { Relevance: }-1 \\
\text { Precision: } 0 \\
\text { Publication } \\
\text { bias: } 0\end{array}$ \\
\hline Quercetin & {$[39]$} & 1 & $\begin{array}{l}\text { Improvement } \\
\text { compared to control }\end{array}$ & Moderate $(+++)$ & $\begin{array}{c}\text { Risk of bias: }-1 \\
\text { Consistency: } 0 \\
\text { Relevance: } 0 \\
\text { Precision: } 0 \\
\text { Publication } \\
\text { bias: } 0\end{array}$ \\
\hline Ubiquinone (Q10) & [40] & 1 & $\begin{array}{l}\text { Improvement } \\
\text { compared to control }\end{array}$ & Moderate $(+++)$ & $\begin{array}{c}\text { Risk of bias: }-1 \\
\text { Consistency: } 0 \\
\text { Relevance: } 0 \\
\text { Precision: } 0 \\
\text { Publication } \\
\text { bias: } 0\end{array}$ \\
\hline \multicolumn{6}{|l|}{ Pro-, pre- and synbiotics } \\
\hline Prebiotics & {$[42]$} & 1 & $\begin{array}{l}\text { Significantly worse } \\
\text { compared to control }\end{array}$ & Low $(++)$ & $\begin{array}{c}\text { Risk of bias: }-2 \\
\text { Consistency: } 0 \\
\text { Relevance: } 0 \\
\text { Precision: } 0 \\
\text { Publication } \\
\text { bias: } 0\end{array}$ \\
\hline $\begin{array}{l}\text { Probiotics and synbiotics, } \\
\text { overall } \\
\text { Probiotics and synbiotics } \\
\text { containing L Casei }\end{array}$ & $\begin{array}{c}{[43-46]} \\
{[43,44,46]}\end{array}$ & $\begin{array}{l}4 \\
3\end{array}$ & $\begin{array}{l}\text { Inconsistent overall } \\
\text { Consistent } \\
\text { improvement } \\
\text { compared to control }\end{array}$ & $\begin{array}{c}\text { Low }(+++) \\
\text { Moderate }(+++)\end{array}$ & $\begin{array}{c}\text { Risk of bias: } 0 \\
\text { Consistency: }-2 \\
\text { Relevance: } 0 \\
\text { Precision: } 0 \\
\text { Publication } \\
\text { bias: } 0 \\
\text { Risk of bias: } 0 \\
\text { Consistency: } 0 \\
\text { Relevance: } 0 \\
\text { Precision: } 0 \\
\text { Publication } \\
\text { bias: }-1\end{array}$ \\
\hline
\end{tabular}




\subsection{Studies on Spices in High Doses}

Three studies investigated the effects of spices as capsules or tablets on DAS28. All three studies were conducted in Iran, by different research groups. The studies included 40-70 participants; all women, except for one study [25] that included 11\% men. Aryaeian et al. compared $1.5 \mathrm{~g} /$ day ginger power in capsules to placebo capsules, in a three month parallel RCT [25]. Shishehbor et al. studied $2 \mathrm{~g} /$ day cinnamon power (Cinnamomum burmannii) in comparison to placebo capsules in a two month RCT [26]. Hamidi et al. compared $100 \mathrm{mg} /$ day of saffron to placebo in a 3 month RCT [27]. All three studies showed significant improvement in DAS28 in comparison to the placebo. Shishehbor et al. and Hamidi et al. both found effects on swollen joints, and the former also found an effect on CRP (Aryaeian et al. did not report results on the individual components of DAS28). The studies were of high [26] and intermediate quality [25,27]. The quality of evidence was considered moderate that daily large doses of cinnamon, ginger, and saffron reduces DAS28 in RA.

\subsection{Studies on n-3 Fatty Acid Supplements}

Five studies investigated the effects of fatty acid supplements for $2.5-4$ months on disease activity in RA. The studies included predominately women. The interventions were heterogeneous and the n-3 fatty acids were delivered in different vehicles. Galaragga et al. provided 97 participants with either placebo or capsules with $10 \mathrm{~g} /$ day cod liver oil for 12 weeks [28]. Das Gupta gave their 100 participants $3 \mathrm{mg} / \mathrm{d}$ of n3-fatty acids in capsules in addition to drug Indomethiacin, while controls received only the drug for 12 weeks [29]. In 2018, Dawcynski et al. provided their 38 participants with either foods enriched with $2.36 \mathrm{mg} / \mathrm{d}$ of n3-fatty acids from a micro algae, or with conventional foods for 10 weeks [30]. In 2009, Dawcynski et al. compared $2.4 \mathrm{~g} /$ day of n3-fatty acids in the form of enriched dairy products to commercial dairy products in 45 patients with RA [31]. Remans et al. gave their 66 participants either a liquid nutritional supplement containing $1.7 \mathrm{~g} / \mathrm{d}$ of n3 PUFA (EPA, DHA, DPA and ALA), other macro- and micronutrients (specified) or a placebo drink [32]. At baseline, disease activity was intermediate or high. Only one [29] of the studies showed a significant effect on DAS28 in comparison to control (effects on swollen and tender joints). The study quality was intermediate for all studies. Overall, the studies showed no effect of $1.7-3 \mathrm{~g} / \mathrm{d}$ of $\mathrm{n}-3$ fatty acids on DAS28 (low quality evidence).

\subsection{Studies on Vitamin D Supplements}

Two studies investigated the effect of vitamin D supplementation on disease activity in RA, in comparison to placebo. The studies included 22-150 participants, predominately women. The study duration was 3 months in one study [33], and 12 months in the other [34]. In both studies, very large doses of vitamin D were given, and the effect was compared to placebo in the control group. Buondonno et al. used a single dose of $7500 \mu \mathrm{g}$ of vitamin D3 (calculated as average per day of follow up, $83 \mu \mathrm{g} / \mathrm{day}$ ) [33]. Hansen et al. intervened with three weekly doses of $1250 \mu \mathrm{g} /$ week of vitamin D2 (corresponding to $180 \mu \mathrm{g} /$ day), followed by two monthly doses of $1250 \mu \mathrm{g}$ (corresponding to $83 \mu \mathrm{g} /$ day) for month $2-11$. Both the intervention group and the control group received $1500 \mathrm{mg} / \mathrm{d}$ of calcium [34]. The baseline disease activity was low in the study by Hansen et al. and high in the study by Buondonno et al. None of the studies found an effect of vitamin D on DAS28, compared to control. Study quality was intermediate [33] and low [34]. Overall, the studies showed no effect of vitamin D supplementation on DAS28 (low quality evidence).

\subsection{Studies on Other Micronutrients}

Rastmanesh et al. compared $6 \mathrm{~g} /$ day of potassium in the form of enriched grape juice to conventional grape juice among 38 hypokalemic women with RA in a parallel study design [35]. The baseline disease activity was high. After one month, there was a significant reduction in DAS28 in the intervention group compared to control (effects on tender and swollen joints, CRP, ESR and general 
health). The quality of the study was considered intermediate. The strength of evidence that potassium in the form of enriched juice reduces DAS28 in hypokalemic women with RA was considered low.

Shishavan et al. randomized 64 female participants with RA in remission either $10 \mu \mathrm{g} /$ day of vitamin $\mathrm{K} 1$ as a chewable tablet, or placebo [36]. After two months, there was no significant difference in DAS28 between the groups. The study was considered to be of high quality. The strength of evidence was considered low that vitamin $\mathrm{K}$ reduce DAS28 in RA.

Van Ede et al. compared $1 \mathrm{mg} /$ day folic acid or $2.5 \mathrm{mg} /$ week of folinic acid, in addition to Methotrexate, to placebo among 434 women (71\%) and men [37]. All three groups received Methotrexate treatment, and folinic or folic acid were given in order to study the effects on efficacy and toxicity. At baseline, disease activity was intermediate. Neither folic nor folinic acid had any effect on DAS28. The study was considered to be of intermediate quality. Based on this study, folic or folinic acid do not reduce DAS28 in RA (moderate quality of evidence).

\subsection{Studies on Single Antioxidants}

Four studies from Iran or Egypt, from different research groups, investigated the effects of single antioxidants on disease activity in RA; in three of the studies the effect was compared to placebo [38-40] and in one the effect was compared to regular treatment [41]. The antioxidant interventions lasted two-three months and comprised of $1200 \mathrm{mg} / \mathrm{d}$ alpha lipolic acid [38], $500 \mathrm{mg} / \mathrm{d}$ quercetin [39], $1 \mathrm{~g} / \mathrm{d}$ resveratrol [41] and $100 \mathrm{mg} / \mathrm{d}$ ubiquinone [40]. There were 50-100 participants per study, predominately women.

Gargari found no effect on DAS28 of alpha lipolic acid, in comparison to placebo, in a study of patients mainly in remission [38]. However, the three other studies found significant improvements in DAS28, compared to placebo, from quercetin [39] (no significant effects on individual components), resveratrol [41] (effect on swollen and tender joints, ESR and CRP) and ubiquinone [40] (effects on swollen and tender joint) in patients with intermediate to high disease activity. The studies were considered to be of high [38] and intermediate quality [39-41]. The strength of evidence that alpha lipolic acid does not reduce disease activity in RA was considered moderate. The quality of evidence for the positive effects on DAS28 in RA was considered moderate for quercetin and ubiquinone, and low for resveratrol.

\subsection{Studies on Pre-, Pro- and Synbiotics}

Five studies investigated the effects of pre-, [42] pro- [43-45] and synbiotics [46] on disease activity in RA. All but two studies were conducted in Iran and two studies were from the same research group [44,46].

Alavi et al. compared a prebiotic supplement providing $1.3 \mathrm{~g} /$ day of fiber (aloe vera gel extract, arabinogalactan, gum ghatti, gum tragacanth, glucosamine) to control (rice flour) in 78 women and men with RA with intermediate disease activity. After six months, DAS28 was significantly worsened in the intervention group, compared to control. However, this difference was due to an improvement in the control group. The study quality was considered low [42].

Alipour et al. compared one daily capsule of L. casei $01\left(10^{8}\right.$ colony forming units $)$ and maltrodextrin to maltrodextrin alone, among 60 women with RA. At baseline, the mean DAS28 indicated remission. After two months, the DAS28-CRP was lower in the intervention group compared to the control group [43] (effects on all individual components of DAS28). Study quality was considered intermediate.

In 2016, Zamani et al. compared a placebo (starch) to probiotic capsules containing L. casei $\left(2 \times 10^{9}\right.$ colony forming units), L. Acidophilus $\left(2 \times 10^{9}\right.$ colony forming units) and B. Bifidum $\left(2 \times 10^{9}\right.$ colony forming units). The study was conducted among 60 women and men for two months. The baseline disease activity was intermediate. After two months, the probiotic intervention reduced DAS 28 in comparison to placebo (effects on CRP) [44]. The study quality was considered high. 
Pineda et al. compared two daily capsules of probiotics containing $2 \times 10^{9}$ of L. Rhamnous and L. Reuteri, in addition to inactive ingredients, to placebo (same ingredients but without the bacteria). The study included 29 patients, both men and women, and the disease activity at baseline was intermediate. After three months, there was no significant difference in disease activity between intervention and control [45]. The quality of the study was intermediate.

In 2017, Zamani et al. compared a synbiotic supplement containing the same probiotics as their previous trial in addition to $800 \mathrm{mg}$ of inulin. The control group received placebo (starch). The study included 54 women and men, and the baseline disease activity was intermediate. After two months, disease activity was significantly reduced in the synbiotic group, in comparison to control (effects on CRP) [46]. The study quality was considered high.

Prebiotic fiber supplementation does not seem to reduce DAS28 in RA (low quality evidence). The strength of evidence was considered low that probiotic supplementation, with and without the addition of prebiotics, reduces DAS28 in RA. The evidence that probiotics containing L. casei improve disease activity compared to control was considered moderate.

\section{Discussion}

This systematic review included 33 RCT studies of diet or dietary supplements that reduce the disease activity of RA. The evidence was considered moderate that a Mediterranean style diet, ginger powder, cinnamon, saffron, quercetin, ubiquinone and probiotics containing L. casei reduce DAS28 in RA. The evidence was considered moderate that cranberry juice, folic acid and alpha lipoic acid do not reduce DAS28 in RA. The evidence for other interventions was very low or low, with only indicative effects. Most findings have never been verified in subsequent trials, making the strength of evidence limited. Consequently, there is a great need to confirm previous positive findings in order to conclude weather diet or dietary supplements can be used as adjuvant treatments to reduce disease activity in RA.

Dietary components can affect disease activity in RA through several direct or indirect interactions with the immune system. Long chain n-3 fatty acids found in fish can decrease the production of inflammatory eicosanoids, adhesion molecules and cytokines [47]. Antioxidants can reduce oxidative stress and thereby possibly symptoms and inflammation in RA [48]. In addition, dietary fiber, prebiotics or probiotics could have beneficial effects on disease activity in RA through modification of the microbiota [49]. Specific nutrients and alterations to microbiota can also affect the intestinal permeability, which may affect the immune response. Lastly, weight loss from dietary intervention could be beneficial for patients with RA, as obesity is associated with the more rapid progression of disabilities [50], but energy deficiency could also put patients at risk of malnutrition [51].

The findings from the current study indicate some positive effects of the Mediterranean diet that combines several of these factors, such as n3-fatty acids, fibre and dietary antioxidants [11]. The null effects from most of the n-3 fatty acid supplement interventions included in the current systematic review suggest that supplementation may be ineffective, despite doses of $n-3$ fatty acids unattainable through diet alone. The supplemented fatty acids will only contribute with a very small proportion of the total daily fat intake. The effect of manipulating the total fat quality of the diet is unclear and warrants further investigation. The findings of this review also show potentially beneficial effects from the supplements containing single antioxidants and spices with high antioxidant capacity. Antioxidant supplementation has been associated with both beneficial and adverse effects in many health conditions [52-54], and the safety of long-term high dose intake is uncertain. The doses of vitamin $\mathrm{D}$ given in the included studies, though ineffective in reducing disease activity, exceed the tolerable upper intake limit set by the European Food Safety Authority [55]. The doses given of spices in the included studies—approximately one teaspoon/day-might be achievable through diet alone. It is however noteworthy that this dose approximately corresponds to the tolerable daily intake for consumption of cinnamon, to avoid a toxic intake of coumarin [56]. Overall, high dose supplementation with nutrients, spices or antioxidants should be initiated with caution as adverse effects are possible. 
Most of the included studies had study durations of two to four months. This seems sufficient to see changes in DAS28, according to the findings. Heterogeneity was observed in regards to which component of DAS28 was affected, as positive effects seemed to be mediated through different factors (inflammation, joint status or self-reported health) in different studies. All studies also included mostly or exclusively female participants, likely due to the overrepresentation of RA among women. However, most of the other aspects of study design, such as baseline disease activity, pharmacological treatment, disease duration, sample size and intervention, varied substantially between studies. These considerable heterogeneities in study design makes it difficult to summarize the results. This was expected, and therefore no meta-analysis was attempted.

A 2009 Cochrane review concluded that the effects of dietary intervention in RA are uncertain due to insufficient evidence [15]. Our conclusions for only DAS28 are similar, thus the evidence has not improved in the last 11 years despite new publications. This is in part due to a lack of significant results in recent dietary intervention trials, but also due to a lack of new dietary intervention studies. A previous systematic review and meta-analysis found no effects on DAS28 of n3-fatty acid supplementation [57], in line with our findings. However, other effects of n3-fatty acid supplementation were observed in the previous review, such as reduced inflammatory cytokines or improved blood lipid profile. Previous systematic reviews have found some indications of positive effects from diet on RA, other than on DAS28. These effects include the positive effects on pain and physical function of the Mediterranean diet [58], and on pain and improvement rates of fasting followed by vegetarian diet [59], but no evidence for effects of $n$-fatty acids or Mediterranean diet on fatigue [60]. The effects of diet on subjective outcomes such as pain and self-reported physical function are not unexpected from interventions that are difficult to blind, and where placebo effects may contribute significantly. Therefore, the relevance of these findings is unclear.

There was moderate strength of evidence for positive effects of probiotics containing L. casei, with or without prebiotics. This contradicts a 2017 meta-analysis that found no effect of probiotics on DAS [61], but with only partially the same studies included. The probiotics used in the studies included in the current systematic review comprised of different combinations of bacteria, but L. casei was used in most. As the results from the studies that used dietary probiotics did not show significant improvements in DAS28 [21,22], supplemental doses may be required. In addition, the bacteria strains in the supplemental studies were different from those in the diet interventions.

\section{Limitations and Strengths}

A limitation of this work is the lack of outcomes other than DAS28. Other outcomes were excluded in order to make a comprehensible summary of results, while still enabling the inclusion of a broad range of interventions. DAS28 was chosen as the outcome as it is the most relevant assessment of disease activity in RA, and is used in clinical praxis. Still, including studies with other indicators of disease activity would have yielded a few more studies, but also would have complicated the interpretation of the results. Including other indicators of disease activity would also have meant the inclusion of mainly older studies. Choosing DAS28 as the outcome ensures results from current, relevant populations that have access to modern pharmacological treatment. Another possible limitation is that we only considered between-group differences in DAS28. However, DAS28 varies over time, and in studies that utilize a minimum threshold for inclusion, regression to the mean is to be expected. Therefore, within-group effects can be due to the natural fluctuations, rather than true effects. Other limitations are associated with the lack of high-quality studies, such as the lack of ITT analysis in the included papers, which means that the effects from single studies are likely inflated. The power was likely insufficient to detect significant effects in some of the studies, though most based their sample size on a priori power calculations. As few experiments have been reproduced, there is also a risk of publication bias where non-significant findings have not been reported. An indication of publication bias is the 13 conference abstracts identified in the searches that never seemed to have resulted in a published paper. 
The strengths of this work include the use of two blinded assessors for article selection, quality assessment and data extraction. When one assessor had co-authored an included paper, a third blinded assessor performed quality assessment to ensure a fair appraisal. In addition, a skilled librarian performed all searches in three databases, safeguarding high quality searches that would include all relevant papers.

\section{Conclusions}

Based on the available results from RCT studies, evidence for the effects of specific diets, food items, spices, micronutrients, antioxidants and pre-, pro- and synbiotics on DAS28 is limited. Positive findings need confirmation in future high-quality studies.

Supplementary Materials: The following are available online at http://www.mdpi.com/2072-6643/12/10/2991/s1, Table S1: Population, Intervention, Control, Outcome (PICO) statement, Table S2: Search strategy.

Author Contributions: The study protocol was developed by L.B. H.S. performed all searches and delivered the results to the assessors. J.N. and L.B. performed data extraction and quality assessment. S.M.U. performed additional quality assessment of studies where L.B. was involved. J.N., H.S., I.G., S.M.U., H.M.L. and L.B. contributed to discussions on which studies to include when uncertain, and to writing the paper. J.N., H.S., I.G., S.M.U., H.M.L. and L.B. All authors have read and agreed to the published version of the manuscript.

Funding: This research received no external funding.

Conflicts of Interest: The authors declare no conflict of interest.

\section{References}

1. Nam, J.L.; Winthrop, K.L.; van Vollenhoven, R.F.; Pavelka, K.; Valesini, G.; Hensor, E.M.; Worthy, G.; Landewe, R.; Smolen, J.S.; Emery, P.; et al. Current evidence for the management of rheumatoid arthritis with biological disease-modifying antirheumatic drugs: A systematic literature review informing the EULAR recommendations for the management of RA. Ann. Rheum. Dis. 2010, 69, 976-986. [CrossRef] [PubMed]

2. Scott, D.L.; Steer, S. The course of established rheumatoid arthritis. Best Pract. Res. Clin. Rheumatol. 2007, 21, 943-967. [CrossRef] [PubMed]

3. Tedeschi, S.K.; Frits, M.; Cui, J.; Zhang, Z.Z.; Mahmoud, T.; Iannaccone, C.; Lin, T.C.; Yoshida, K.; Weinblatt, M.E.; Shadick, N.A.; et al. Diet and Rheumatoid Arthritis Symptoms: Survey Results From a Rheumatoid Arthritis Registry. Arthritis Care Res. 2017, 69, 1920-1925. [CrossRef] [PubMed]

4. Badsha, H. Role of Diet in Influencing Rheumatoid Arthritis Disease Activity. Open Rheumatol. J. 2018, 12, 19-28. [CrossRef]

5. Oliviero, F.; Scanu, A.; Zamudio-Cuevas, Y.; Punzi, L.; Spinella, P. Anti-inflammatory effects of polyphenols in arthritis. J. Sci. Food Agric. 2018, 98, 1653-1659. [CrossRef] [PubMed]

6. Colotta, F.; Jansson, B.; Bonelli, F. Modulation of inflammatory and immune responses by vitamin D. J. Autoimmun. 2017, 85, 78-97. [CrossRef] [PubMed]

7. Chiang, E.P.I.; Bagley, P.J.; Selhub, J.; Nadeau, M.; Roubenoff, R. Abnormal vitamin B6 status is associated with severity of symptoms in patients with rheumatoid arthritis. Am. J. Med. 2003, 114, 283-287. [CrossRef]

8. Sahebari, M.; Ayati, R.; Mirzaei, H.; Sahebkar, A.; Hejazi, S.; Saghafi, M.; Saadati, N.; Ferns, G.A.; Ghayour-Mobarhan, M. Serum Trace Element Concentrations in Rheumatoid Arthritis. Biol. Trace Elem. Res. 2016, 171, 237-245. [CrossRef]

9. Yadav, H.; Lee, J.H.; Lloyd, J.; Walter, P.; Rane, S.G. Beneficial metabolic effects of a probiotic via butyrate-induced GLP-1 hormone secretion. J. Biol. Chem. 2013, 288, 25088-25097. [CrossRef]

10. Homayouni Rad, A.; Torab, R.; Ghalibaf, M.; Norouzi, S.; Mehrabany, E.V. Might patients with immune-related diseases benefit from probiotics? Nutrition 2013, 29, 583-586. [CrossRef]

11. Davis, C.; Bryan, J.; Hodgson, J.; Murphy, K. Definition of the mediterranean diet: A literature review. Nutrients 2015, 7, 9139-9153. [CrossRef] [PubMed]

12. Ramezani-Jolfaie, N.; Mohammadi, M.; Salehi-Abargouei, A. The effect of healthy Nordic diet on cardio-metabolic markers: A systematic review and meta-analysis of randomized controlled clinical trials. Eur. J. Nutr. 2018, 58, 2159-2174. [CrossRef] [PubMed] 
13. Koebnick, C.; Garcia, A.L.; Dagnelie, P.C.; Strassner, C.; Lindemans, J.; Katz, N.; Leitzmann, C.; Hoffmann, I. Long-term consumption of a raw food diet is associated with favorable serum LDL cholesterol and triglycerides but also with elevated plasma homocysteine and low serum HDL cholesterol in humans. J. Nutr. 2005, 135, 2372-2378. [CrossRef] [PubMed]

14. Davey, G.K.; Spencer, E.A.; Appleby, P.N.; Allen, N.E.; Knox, K.H.; Key, T.J. EPIC-Oxford: Lifestyle characteristics and nutrient intakes in a cohort of 33883 meat-eaters and 31546 non meat-eaters in the UK. Public Health Nutr. 2003, 6, 259-269. [CrossRef]

15. Hagen, K.B.; Byfuglien, M.G.; Falzon, L.; Olsen, S.U.; Smedslund, G. Dietary interventions for rheumatoid arthritis. Cochrane Database Syst. Rev. 2009, 21, CD006400. [CrossRef]

16. Moher, D.; Liberati, A.; Tetzlaff, J.; Altman, D.G. Preferred reporting items for systematic reviews and meta-analyses: The PRISMA statement. PLoS Med. 2009, 6, e1000097. [CrossRef] [PubMed]

17. Fransen, J.; Stucki, G.; van Riel, P.L.C.M. Rheumatoid arthritis measures: Disease Activity Score (DAS), Disease Activity Score-28 (DAS28), Rapid Assessment of Disease Activity in Rheumatology (RADAR), and Rheumatoid Arthritis Disease Activity Index (RADAI). Arthritis Care Res. 2003, 49, S214-S224. [CrossRef]

18. NNR5 Working Group. A Guide for Conducting Systematic Literature Reviews for the 5th Edition of the Nordic Nutrition Recommendations, Revised ed.; Nordic Council of Ministers: Copenhagen, Denmark, 2011. Available online: http://www.slv.se/upload/NNR5/A\%20guide $\% 20$ for $\% 20$ conducting\%20SLR\%20for $\%$ 20NNR5\%20FINAL.pdf (accessed on 5 July 2019).

19. Atkins, D.; Best, D.; Briss, P.A.; Eccles, M.; Falck-Ytter, Y.; Flottorp, S.; Guyatt, G.H.; Harbour, R.T.; Haugh, M.C.; Henry, D.; et al. Grading quality of evidence and strength of recommendations. BMJ 2004, 328, 1490.

20. Skoldstam, L.; Hagfors, L.; Johansson, G. An experimental study of a Mediterranean diet intervention for patients with rheumatoid arthritis. Ann. Rheum. Dis. 2003, 62, 208-214. [CrossRef]

21. Nenonen, M.T.; Helve, T.A.; Rauma, A.L.; Hanninen, O.O. Uncooked, lactobacilli-rich, vegan food and rheumatoid arthritis. Br. J. Rheumatol. 1998, 37, 274-281. [CrossRef]

22. Vadell, A.K.E.; Bärebring, L.; Hulander, E.; Gjertsson, I.; Lindqvist, H.M.; Winkvist, A. Anti-inflammatory Diet In Rheumatoid Arthritis (ADIRA)-a randomized, controlled crossover trial indicating effects on disease activity. Am. J. Clin. Nutr. 2020, 111, 1203-1213. [CrossRef] [PubMed]

23. Thimóteo, N.S.B.; Iryioda, T.M.V.; Alfieri, D.F.; Rego, B.E.F.; Scavuzzi, B.M.; Fatel, E.; Lozovoy, M.A.B.; Simão, A.N.C.; Dichi, I. Cranberry juice decreases disease activity in women with rheumatoid arthritis. Nutrition 2019, 60, 112-117. [CrossRef] [PubMed]

24. Lindqvist, H.M.; Gjertsson, I.; Eneljung, T.; Winkvist, A. Influence of Blue Mussel (Mytilus edulis) Intake on Disease Activity in Female Patients with Rheumatoid Arthritis: The MIRA Randomized Cross-Over Dietary Intervention. Nutrients 2018, 10, 481. [CrossRef] [PubMed]

25. Aryaeian, N.; Shahram, F.; Mahmoudi, M.; Tavakoli, H.; Yousefi, B.; Arablou, T.; Jafari Karegar, S. The effect of ginger supplementation on some immunity and inflammation intermediate genes expression in patients with active Rheumatoid Arthritis. Gene 2019, 698, 179-185. [CrossRef] [PubMed]

26. Shishehbor, F.; Rezaeyan Safar, M.; Rajaei, E.; Haghighizadeh, M.H. Cinnamon Consumption Improves Clinical Symptoms and Inflammatory Markers in Women With Rheumatoid Arthritis. J. Am. Coll. Nutr. 2018, 37, 685-690. [CrossRef]

27. Hamidi, Z.; Aryaeian, N.; Abolghasemi, J.; Shirani, F.; Hadidi, M.; Fallah, S.; Moradi, N. The effect of saffron supplement on clinical outcomes and metabolic profiles in patients with active rheumatoid arthritis: A randomized, double-blind, placebo-controlled clinical trial. Phytother. Res. 2020, 34, 1650-1658. [CrossRef]

28. Galarraga, B.; Ho, M.; Youssef, H.M.; Hill, A.; McMahon, H.; Hall, C.; Ogston, S.; Nuki, G.; Belch, J.J. Cod liver oil (n-3 fatty acids) as an non-steroidal anti-inflammatory drug sparing agent in rheumatoid arthritis. Rheumatology 2008, 47, 665-669. [CrossRef]

29. Das Gupta, A.B.; Hossain, A.K.; Islam, M.H.; Dey, S.R.; Khan, A.L. Role of omega-3 fatty acid supplementation with indomethacin in suppression of disease activity in rheumatoid arthritis. Bangladesh Med. Res. Counc. Bull. 2009, 35, 63-68. [CrossRef]

30. Dawczynski, C.; Dittrich, M.; Neumann, T.; Goetze, K.; Welzel, A.; Oelzner, P.; Völker, S.; Schaible, A.M.; Troisi, F.; Thomas, L.; et al. Docosahexaenoic acid in the treatment of rheumatoid arthritis: A double-blind, placebo-controlled, randomized cross-over study with microalgae vs. sunflower oil. Clin. Nutr. 2018, 37, 494-504. [CrossRef] 
31. Dawczynski, C.; Schubert, R.; Hein, G.; Müller, A.; Eidner, T.; Vogelsang, H.; Basu, S.; Jahreis, G. Long-term moderate intervention with n-3 long-chain PUFA-supplemented dairy products: Effects on pathophysiological biomarkers in patients with rheumatoid arthritis. Br. J. Nutr. 2009, 101, 1517-1526. [CrossRef]

32. Remans, P.H.; Sont, J.K.; Wagenaar, L.W.; Wouters-Wesseling, W.; Zuijderduin, W.M.; Jongma, A.; Breedveld, F.C.; Van Laar, J.M. Nutrient supplementation with polyunsaturated fatty acids and micronutrients in rheumatoid arthritis: Clinical and biochemical effects. Eur. J. Clin. Nutr. 2004, 58, 839-845. [CrossRef] [PubMed]

33. Buondonno, I.; Rovera, G.; Sassi, F.; Rigoni, M.M.; Lomater, C.; Parisi, S.; Pellerito, R.; Isaia, G.C.; D'Amelio, P. Vitamin D and immunomodulation in early rheumatoid arthritis: A randomized double-blind placebo-controlled study. PLOS ONE 2017, 12, e0178463. [CrossRef] [PubMed]

34. Hansen, K.E.; Bartels, C.M.; Gangnon, R.E.; Jones, A.N.; Gogineni, J. An evaluation of high-dose vitamin D for rheumatoid arthritis. J. Clin. Rheumatol. 2014, 20, 112-114. [CrossRef] [PubMed]

35. Rastmanesh, R.; Abargouei, A.S.; Shadman, Z.; Ebrahimi, A.A.; Weber, C.E. A pilot study of potassium supplementation in the treatment of hypokalemic patients with rheumatoid arthritis: A randomized, double-blinded, placebo-controlled trial. J. Pain 2008, 9, 722-731. [CrossRef]

36. Shishavan, N.G.; Gargari, B.P.; Jafarabadi, M.A.; Kolahi, S.; Haggifar, S.; Noroozi, S. Vitamin K(1) Supplementation Did Not Alter Inflammatory Markers and Clinical Status in Patients with Rheumatoid Arthritis. Int. J. Vitam. Nutr. Res. 2018, 88, 251-257. [CrossRef]

37. van Ede, A.E.; Laan, R.F.; Rood, M.J.; Huizinga, T.W.; van de Laar, M.A.; van Denderen, C.J.; Westgeest, T.A.; Romme, T.C.; de Rooij, D.J.; Jacobs, M.J.; et al. Effect of folic or folinic acid supplementation on the toxicity and efficacy of methotrexate in rheumatoid arthritis: A forty-eight week, multicenter, randomized, double-blind, placebo-controlled study. Arthritis Rheum. 2001, 44, 1515-1524. [CrossRef]

38. Gargari, B.P.; Kolahi, S.; Dehghan, P.; Khabbazi, A.; Mirtaheri, E. Effects of alpha-lipoic acid supplementation on clinical status and anthropometric indices in women with rheumatoid arthritis. Curr. Top. Nutraceutical Res. 2015, 13, 33-40.

39. Javadi, F.; Ahmadzadeh, A.; Eghtesadi, S.; Aryaeian, N.; Zabihiyeganeh, M.; Rahimi Foroushani, A.; Jazayeri, S. The Effect of Quercetin on Inflammatory Factors and Clinical Symptoms in Women with Rheumatoid Arthritis: A Double-Blind, Randomized Controlled Trial. J. Am. Coll. Nutr. 2017, 36, 9-15. [CrossRef]

40. Nachvak, S.M.; Alipour, B.; Mahdavi, A.M.; Aghdashi, M.A.; Abdollahzad, H.; Pasdar, Y.; Samadi, M.; Mostafai, R. Effects of coenzyme Q10 supplementation on matrix metalloproteinases and DAS-28 in patients with rheumatoid arthritis: A randomized, double-blind, placebo-controlled clinical trial. Clin. Rheumatol. 2019, 38, 3367-3374. [CrossRef]

41. Khojah, H.M.; Ahmed, S.; Abdel-Rahman, M.S.; Elhakeim, E.H. Resveratrol as an effective adjuvant therapy in the management of rheumatoid arthritis: A clinical study. Clin. Rheumatol. 2018, 37, 2035-2042. [CrossRef]

42. Alavi, A.; Goodfellow, L.; Fraser, O.; Tarelli, E.; Bland, M.; Axford, J. A double-blind, randomized, placebo-controlled study to explore the efficacy of a dietary plant-derived polysaccharide supplement in patients with rheumatoid arthritis. Rheumatology 2011, 50, 1111-1119. [CrossRef]

43. Alipour, B.; Homayouni-Rad, A.; Vaghef-Mehrabany, E.; Sharif, S.K.; Vaghef-Mehrabany, L.; Asghari-Jafarabadi, M.; Nakhjavani, M.R.; Mohtadi-Nia, J. Effects of Lactobacillus casei supplementation on disease activity and inflammatory cytokines in rheumatoid arthritis patients: A randomized double-blind clinical trial. Int. J. Rheum. Dis. 2014, 17, 519-527.

44. Zamani, B.; Golkar, H.R.; Farshbaf, S.; Emadi-Baygi, M.; Tajabadi-Ebrahimi, M.; Jafari, P.; Akhavan, R.; Taghizadeh, M.; Memarzadeh, M.R.; Asemi, Z. Clinical and metabolic response to probiotic supplementation in patients with rheumatoid arthritis: A randomized, double-blind, placebo-controlled trial. Int. J. Rheum. Dis. 2016, 19, 869-879. [CrossRef] [PubMed]

45. Pineda Mde, L.; Thompson, S.F.; Summers, K.; de Leon, F.; Pope, J.; Reid, G. A randomized, double-blinded, placebo-controlled pilot study of probiotics in active rheumatoid arthritis. Med. Sci. Monit. 2011, 17, CR347-CR354.

46. Zamani, B.; Farshbaf, S.; Golkar, H.R.; Bahmani, F.; Asemi, Z. Synbiotic supplementation and the effects on clinical and metabolic responses in patients with rheumatoid arthritis: A randomised, double-blind, placebo-controlled trial. Br. J. Nutr. 2017, 117, 1095-1102. [CrossRef] [PubMed] 
47. Calder, P.C. Polyunsaturated fatty acids and inflammation. Prostaglandins Leukot. Essent. Fatty Acids 2006, 75, 197-202. [CrossRef] [PubMed]

48. Phull, A.R.; Nasir, B.; Haq, I.U.; Kim, S.J. Oxidative stress, consequences and ROS mediated cellular signaling in rheumatoid arthritis. Chem. Biol. Interact. 2018, 281, 121-136. [CrossRef]

49. Guerreiro, C.S.; Calado, Â.; Sousa, J.; Fonseca, J.E. Diet, Microbiota, and Gut Permeability-The Unknown Triad in Rheumatoid Arthritis. Frontiers Med. 2018, 5, 349. [CrossRef]

50. Baker, J.F.; England, B.R.; Mikuls, T.R.; Sayles, H.; Cannon, G.W.; Sauer, B.C.; George, M.D.; Caplan, L.; Michaud, K. Obesity, Weight Loss, and Progression of Disability in Rheumatoid Arthritis. Arthritis Care Res. 2018, 70, 1740-1747. [CrossRef]

51. Smedslund, G.; Byfuglien, M.G.; Olsen, S.U.; Hagen, K.B. Effectiveness and safety of dietary interventions for rheumatoid arthritis: A systematic review of randomized controlled trials. J. Am. Diet. Assoc. 2010, 110, 727-735. [CrossRef]

52. Salehi, B.; Mishra, A.P.; Nigam, M.; Sener, B.; Kilic, M.; Sharifi-Rad, M.; Fokou, P.V.T.; Martins, N.; Sharifi-Rad, J. Resveratrol: A Double-Edged Sword in Health Benefits. Biomedicines 2018, 6, 91. [CrossRef]

53. Klein, E.A.; Thompson, I.M., Jr.; Tangen, C.M.; Crowley, J.J.; Lucia, M.S.; Goodman, P.J.; Minasian, L.M.; Ford, L.G.; Parnes, H.L.; Gaziano, J.M.; et al. Vitamin E and the risk of prostate cancer: The Selenium and Vitamin E Cancer Prevention Trial (SELECT). JAMA 2011, 306, 1549-1556. [CrossRef] [PubMed]

54. Dutra, M.T.; Alex, S.; Mota, M.R.; Sales, N.B.; Brown, L.E.; Bottaro, M. Effect of strength training combined with antioxidant supplementation on muscular performance. Appl. Physiol. Nutr. Metab. 2018, 43, 775-781. [CrossRef] [PubMed]

55. EFSA Panel on Dietetic Products, Nutrition and Allergies (NDA). Scientific Opinion on the Tolerable Upper Intake Level of vitamin D. EFSA J. 2012, 10, 2813.

56. European Food Safety Authority (EFSA). Coumarin in flavourings and other food ingredients with flavouring properties. EFSA J. 2008, 793, 1-15.

57. Gioxari, A.; Kaliora, A.C.; Marantidou, F.; Panagiotakos, D.P. Intake of $\omega-3$ polyunsaturated fatty acids in patients with rheumatoid arthritis: A systematic review and meta-analysis. Nutrition 2018, 45, 114-124 e4. [CrossRef]

58. Forsyth, C.; Kouvari, M.; D’Cunha, N.M.; Georgousopoulou, E.N.; Panagiotakos, D.B.; Mellor, D.D.; Kellett, J.; Naumovski, N. The effects of the Mediterranean diet on rheumatoid arthritis prevention and treatment: A systematic review of human prospective studies. Rheumatol. Int. 2018, 38, 737-747. [CrossRef]

59. Müller, H.; de Toledo, F.W.; Resch, K.L. Fasting followed by vegetarian diet in patients with rheumatoid arthritis: A systematic review. Scand. J. Rheumatol. 2001, 30,1-10.

60. Cramp, F.; Hewlett, S.; Almeida, C.; Kirwan, J.R.; Choy, E.H.; Chalder, T.; Pollock, J.; Christensen, R. Non-pharmacological interventions for fatigue in rheumatoid arthritis. Cochrane Database Syst. Rev. 2013, 23, CD008322. [CrossRef]

61. Mohammed, A.T.; Khattab, M.; Ahmed, A.M.; Turk, T.; Sakr, N.; Khalil, A.M.; Abdelhalim, M.; Sawaf, B.; Hirayama, K.; Huy, N.T. The therapeutic effect of probiotics on rheumatoid arthritis: A systematic review and meta-analysis of randomized control trials. Clin. Rheumatol. 2017, 36, 2697-2707. [CrossRef]

(C) 2020 by the authors. Licensee MDPI, Basel, Switzerland. This article is an open access article distributed under the terms and conditions of the Creative Commons Attribution (CC BY) license (http://creativecommons.org/licenses/by/4.0/). 